\title{
Influence of sex on intracellular calcium homoeostasis in patients with atrial fibrillation
}

\author{
Adela Herraiz-Martínez ${ }^{1,2 \dagger}$, Carmen Tarifa ${ }^{1,2 \dagger}$, Verónica Jiménez-Sábado ${ }^{2,3 \dagger}{ }^{2,}$, \\ Anna Llach $\mathbb{1}^{2}$, Hector Godoy-Marín ${ }^{4,5}$, Hildegard Colino-Lage $\mathbb{1}^{1,2}$, \\ Carme Nolla-Colomer (1) ${ }^{6}$, Sergi Casabella-Ramon (1) 1,2, Paloma Izquierdo-Castro (1) 1,2, \\ Iván Benítez $\mathbb{1}^{7}{ }^{7}$, Raul Benítez (1) ${ }^{6}$, Elena Roselló-Díez ${ }^{8}{ }^{8,9}$, Enrique Rodríguez-Font ${ }^{10}$, \\ Xavier Viñolas $\mathbb{1}^{10}$, Francisco Ciruela $\mathbb{1}^{4,5}$, Juan Cinca ${ }^{3,9,10}$, and \\ Leif Hove-Madsen (1) 1,2,3*
}

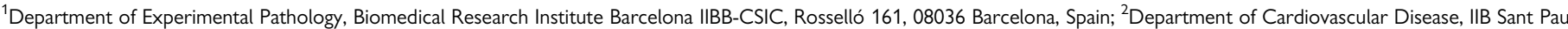
St. Antoni Ma. Claret 167, 08025 Barcelona, Spain; ${ }^{3}$ CIBERCV, Instituto de Salud Carlos III, Pabellón 11, Monforte de Lemos 3-5, 28029 Madrid, Spain; ${ }^{4}$ Neuropharmacology and Pain

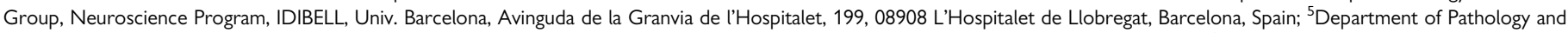

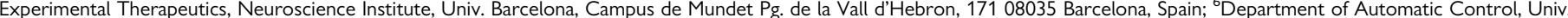
Politècnica de Catalunya, Campus Diagonal Sud, Building H. Av. Diagonal, 64708028 Barcelona, Spain; ${ }^{7}$ Biostatistic Unit, Translational Research in Respiratory Medicine, Biomedical

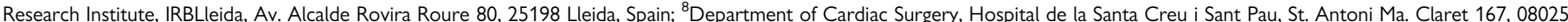

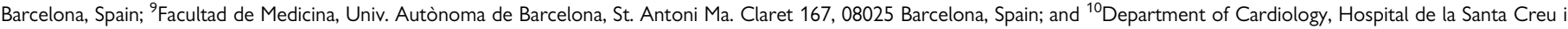
Sant Pau, St. Antoni Ma. Claret 167, 08025 Barcelona, Spain

Received 21 October 2020; editorial decision 24 March 2021; accepted 30 March 2021; online publish-ahead-of-print 31 March 2021

Time for primary review: 16 days

Aims

Atrial fibrillation (AF) has been associated with intracellular calcium disturbances in human atrial myocytes, but little is known about the potential influence of sex and we here aimed to address this issue.

Methods

and results

Alterations in calcium regulatory mechanisms were assessed in human atrial myocytes from patients without AF or with long-standing persistent or permanent AF. Patch-clamp measurements revealed that L-type calcium current $\left(I_{C_{a}}\right)$ density was significantly smaller in males with than without $\mathrm{AF}(-1.15 \pm 0.37 \mathrm{vs}$. $-2.06 \pm 0.29 \mathrm{pA} / \mathrm{pF})$ but not in females with $\mathrm{AF}(-1.88 \pm 0.40$ vs. $-2.21 \pm 0.0 .30 \mathrm{pA} / \mathrm{pF})$. In contrast, transient inward currents $\left(I_{\mathrm{Ti}_{\mathrm{i}}}\right)$ were more frequent in females with than without $A F(1.92 \pm 0.36$ vs. $1.10 \pm 0.19$ events/min) but not in males with $A F$. Moreover, confocal calcium imaging showed that females with AF had more calcium spark sites than those without $\mathrm{AF}\left(9.8 \pm 1.8\right.$ vs. $2.2 \pm 1.9$ sites $\left./ \mu \mathrm{m}^{2}\right)$ and sparks were wider $(3.0 \pm 0.3$ vs. $2.2 \pm 0.3 \mu \mathrm{m})$ and lasted longer ( $79 \pm 6$ vs. $55 \pm 8 \mathrm{~ms}$ ), favouring their fusion into calcium waves that triggers $I_{T \mid} s$ and afterdepolarizations. This was linked to higher ryanodine receptor phosphorylation at $s 2808$ in women with AF, and inhibition of adenosine $A_{2 A}$ or beta-adrenergic receptors that modulate $s 2808$ phosphorylation was able to reduce the higher incidence of $I_{\mathrm{TI}}$ in women with AF.

Conclusion

Perturbations of the calcium homoeostasis in AF is sex-dependent, concurring with increased spontaneous SR calcium release-induced electrical activity in women but not in men, and with diminished $I_{\mathrm{Ca}}$ density in men only.

\footnotetext{
* Corresponding author. Tel: +34 935565620; fax: +34 935565603, E-mail: leif.hove@iibb.csic.es

† These authors contributed equally to this article.

C The Author(s) 2021. Published by Oxford University Press on behalf of the European Society of Cardiology.

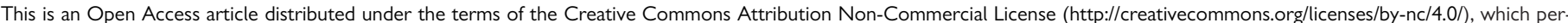
mits non-commercial re-use, distribution, and reproduction in any medium, provided the original work is properly cited. For commercial re-use, please contact journals.permissions@oup.com
} 


\section{Graphical Abstract}

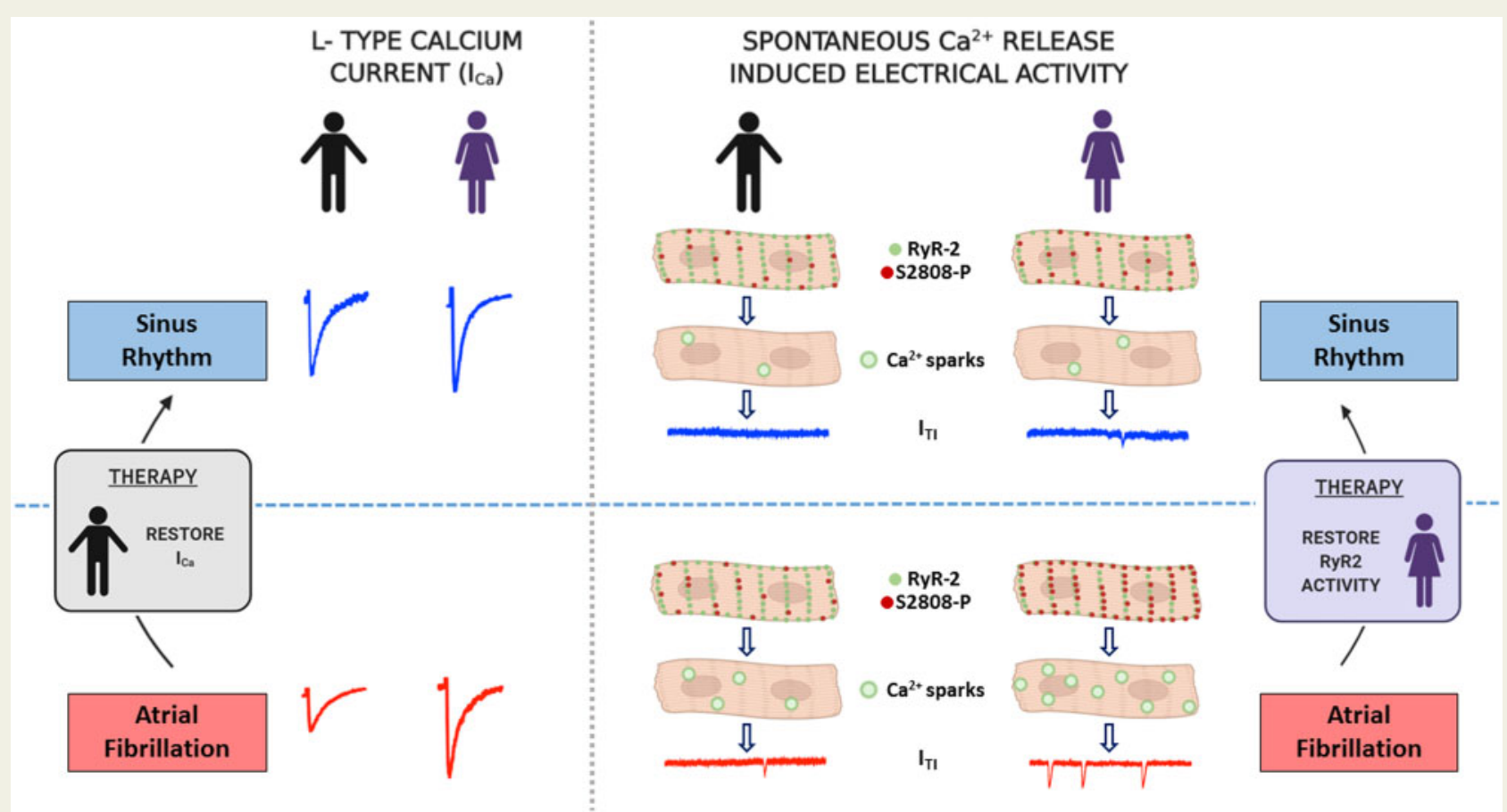

Keywords

Sarcoplasmic reticulum calcium release • Calcium sparks • Transient inward current • Afterdepolarizations • Ryanodine receptor phosphorylation

\section{Introduction}

Epidemiological studies on atrial fibrillation (AF) show that compared with men, women have a lower incidence and a 5-year delay in the onset of this arrhythmia. ${ }^{1-3}$ However, because the incidence of $A F$ increases exponentially at advanced age and because women live longer, the number converges for the two sexes at 75 years or older. ${ }^{3,4}$ The mechanism by which the incidence of $A F$ increases more strongly in women older than 70 years is not well known. Since both AF and sex have been associated with well-defined cellular electrophysiological alterations, ${ }^{5,6}$ it is conceivable that some of them contribute to the sex-related differences in the incidence of AF.

Mechanistically, AF has been associated with both structural ${ }^{7}$ and electrophysiological alterations. ${ }^{5,8}$ Among the electrophysiological alterations, AF has been linked to disturbances in the intracellular calcium homoeostasis $^{9-12}$ including malfunctioning of the sarcoplasmic reticulum $(S R)^{9,10,12-15}$ in experimental series with a mixed population of male and female patients. These studies have shown that atrial myocytes from patients with AF have a reduced L-type calcium current $\left(I_{C_{a}}\right)$ density, ${ }^{10,11}$ which shortens the action potential, and hence reduces the duration of the refractory period. In addition, these myocytes display a high incidence of spontaneous SR calcium release, ${ }^{9}$ which can induce arrhythmogenic spontaneous membrane depolarizations. ${ }^{15}$

Therefore, this study aimed to determine the specific influence of sex on the intracellular calcium homoeostasis in a large series of patients with and without AF.

\section{Methods}

\subsection{Human biological samples and atrial myocyte isolation}

Human right atrial myocytes were isolated from patients undergoing cardiac surgery as previously described ${ }^{9}$ and used for different experimental protocols. Because the yield of myocyte isolation from human atrial samples is variable, not all protocols were carried out in all patients as outlined in the flow scheme for collection and processing of human atrial samples (Supplementary material online, Figure S1). For electrophysiological experimentation, patients were divided into males $(n=189)$ and females $(n=78)$ without AF (237) and with permanent or long-standing persistent AF (30) and key clinical and echocardiographic data as well as pharmacological treatments included in the statistical analysis of this data are summarized in Table 1. Patients undergoing mitral valve replacement or repair were not included in this analysis to avoid potentially confounding effects of mitral valve disease, a disease that already alters the calcium homoeostasis in patients without AF. ${ }^{16}$ Each patient gave written consent to obtain a sample from the right atrial appendage that would otherwise have been discarded during the surgical intervention. The study was approved by the Ethics Committee at Hospital de la Santa Creu i Sant Pau, Barcelona, Spain and the investigation conforms to the principles outlined in the Declaration of Helsinki.

\subsection{Immunoblot and immunofluorescent labelling}

Expression of SERCA2a, Na-Ca exchanger (NCX-1), phospholamban (PLB), PLB phosphorylated at ser-16 (PLB-s16) and PLB phosphorylated 
Table I Clinical characteristics of the study population for electrophysiological analysis

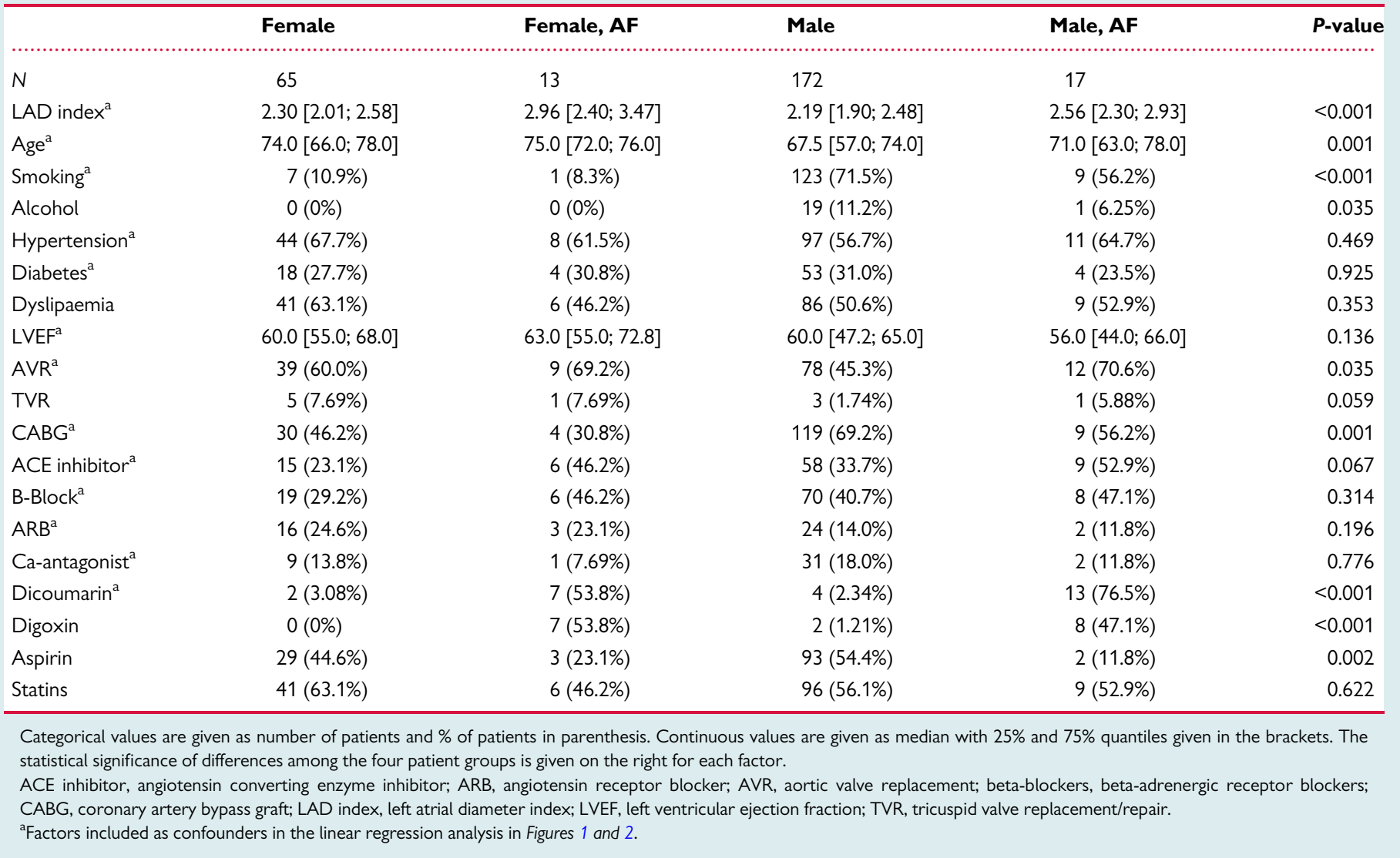

at thr-17 (PLB-t17) was determined by western blot. Atrial samples were pulverized in liquid nitrogen and homogenized in $50 \mathrm{mM}$ Tris- $\mathrm{HCl}$ $\mathrm{pH} 7.4$ containing protease inhibitors. Proteins $(10 \mu \mathrm{g})$ were separated by $5 \%$ or $10 \%$ sodium dodecyl sulphate-polyacrylamide gel electrophoresis (transferred to polyvinylidene difluoride membranes using a semidry transfer system, and immunoblotted using rabbit anti-SERCA2a, rabbit anti-Csq-2, rabbit anti-NCX-1, rabbit anti-PLB, rabbit anti-PLB-s16, rabbit anti-PLB-t17, and rabbit anti- $\alpha$-actinin antibodies. Details are provided in the Supplementary material online, Methods.

Isolated myocytes were fixed with $5 \%$ paraformaldehyde for $10 \mathrm{~min}$ at room temperature. Subsequently, the ryanodine receptor (RyR2), the Ltype calcium channel (Cav1.2), or calsequestrin-2 (Csq-2) were labelled using the primary antibodies mouse anti-RyR2, guinea-pig anti-Cav1.2, rabbit anti-Csq-2, rabbit anti-s2808-P, or rabbit anti-ser2814P as previously described. ${ }^{17}$ After labelling, cells were stored at $4^{\circ} \mathrm{C}$ until the labelled proteins were visualized using confocal microscopy (Supplementary material online, Methods for further details).

\subsection{Patch-clamp technique}

Electrophysiological recordings were performed using perforated patchclamp technique in isolated human atrial myocytes as described in the Supplementary material online, Methods. Briefly, $I_{\mathrm{Ca}}$, spontaneous transient inward calcium release-induced $\mathrm{NCX}$ currents $\left(I_{\mathrm{TI}}\right)$, and the caffeine releasable SR calcium content were measured using whole cell voltage-clamp configuration. Membrane potentials were recorded in the current-clamp configuration. Adenosine $A_{2 A}$ receptors $\left(A_{2 A} R s\right)$ were stimulated with the selective agonist CGS21680 (200 nM). Adenosine deaminase (ADA) with and activity of $2 \mu \mathrm{mol} / \mathrm{min} / \mathrm{mL}$ was used to prevent $A_{2 A} R$ activation.

\subsection{Confocal calcium imaging}

To visualize changes in the intracellular calcium concentration, myocytes were loaded with $2.5 \mu \mathrm{M}$ fluo-4 AM for $15 \mathrm{~min}$, followed by wash and de-esterification for $30 \mathrm{~min}$ or more. Confocal calcium images $(512 \times 140$ pixels) were recorded at a frame rate of $90 \mathrm{~Hz}$, using a resonance-scanning confocal microscope with a $63 \times$ glycerol-immersion objective (Leica SP5 AOBS, Wetzlar, Germany). The excitation wavelength was $488 \mathrm{~nm}$ and fluorescence emission was collected between 500 and $650 \mathrm{~nm}$ with a Leica Hybrid Detector. Experiments were performed at room temperature and calcium sparks were detected using custommade algorithms implemented using MATLAB (The Mathworks Inc., Boston, MA) as previously described. ${ }^{17}$

\subsection{Data analysis and representation}

Experiments were performed without knowledge about clinical data and clinicians gathering the clinical data did not know the experimental results. Data was represented in figures using Excel, Power Point, IGOR Pro or Biorender.com Software. Unless otherwise stated, values were determined for each patient (multiple determinations in the same patient were averaged) and expressed as mean $\pm 95 \%$ confidence level. Where indicated, statistical significance was evaluated using a multivariate linear regression model for data with a normal distribution $\left(I_{C_{a}}\right)$ or generalized linear model with a Poisson distribution $\left(I_{T 1}\right)$. To validate the robustness of this analysis, results were analysed using (i) an unadjusted linear regression model with interaction between sex and atrial rhythm, (ii) a fully 
adjusted model taking into account the confounding effects of common clinical factors showing a bias between sex as well as factors suspected to affect calcium homoeostasis, and (iii) a model adjusted for the most relevant factors (see Supplementary material online, Methods for details). As shown in Supplementary material online, Figure S2, raw data and estimates using the fully adjusted model were similar and the three linear regression models yielded similar results. In the subsequent mechanistic analysis data were analysed using a linear regression model as indicated. Otherwise, Fisher's exact test was used for categorical data. Student's $t$-test or Wilcoxon's rank test was used for paired or unpaired comparisons. The $P$-value threshold defining statistical significance was set at $<0.05$. Statistical analysis was performed using version 3.5.2 of $R$ software.

\section{Results}

\subsection{Differences in clinical characteristics among male and female patients}

Reduced L-type calcium current $\left(I_{C_{a}}\right)$ density ${ }^{11}$ and a higher incidence of $I_{\text {TI }}$ currents $^{9,10,15}$ are cellular electrophysiological hallmarks of atrial myocytes from patients with AF that might be influenced by sex. Therefore, we first determined if there were sex-dependent differences in cardiovascular risk factors; concurrent cardiovascular disease; echocardiographic data; pharmacological treatments or atrial rhythm. Table 1 shows that female patients were older, smoked less, and did not abuse alcohol. There were no significant differences in common pharmacological treatments, but coronary artery bypass grafts were performed in a significantly smaller fraction of women. To exclude potentially confounding effects of these sex-dependent differences in the study population, or differences in the effect of the clinical factors on calcium homoeostasis, we performed a multivariate regression analysis (see Supplementary material online, Methods) in order to determine the specific effects of sex and $\mathrm{AF}$ on the electrophysiological measurements.

\subsection{Effect of sex on L-type calcium current and transient inward currents}

In line with previous findings, comparison of the $I_{\mathrm{Ca}}$ amplitude measured in patients with and without $A F$ showed that those with $A F$ have significantly smaller amplitude $(-1.20 \pm 0.21$ vs. $-2.03 \pm 0.10 \mathrm{pA} / \mathrm{pF}, P<0.001$, unpaired $t$-test). However, multivariate regression analysis taking confounding factors into account (Supplementary material online, Methods) revealed no significant interactions between AF, sex or AF plus sex and $I_{C a}$ density, but pairwise comparisons showed a significant reduction in $I_{C a}$ density in male but not in female patients with $A F$ (Figure $1 A$ and $B$ ). Neither sex nor AF affected the shape of the current-voltage relationship in our patients (Figure 1C), but the time-dependent decay of the $I_{\mathrm{Ca}}$ was slowed significantly in male patients with AF (Figure 1D). Immunofluorescent labelling revealed that the density of L-type calcium channels was higher at the sarcolemma than in the cell centre. However, the difference in $I_{\mathrm{Ca}}$ amplitude among patients with and without AF was not due to differences in the density of the L-type calcium channels among patients with and without AF (Supplementary material online, Figure S3).

Multivariate analysis of the $I_{T I}$ frequency revealed a significant interaction between sex and $I_{T 1}$ frequency $(P<0.001)$ and between sex plus AF and $I_{T I}$ frequency $(P=0.032)$. Hence, $I_{T I}$ frequency was significantly higher in female than in male patients $(P<0.001)$. Moreover, the $I_{T I}$ frequency was low and virtually identical in male patients with and without $A F$ while female patients displayed a significantly higher frequency in those with $A F$ than without AF (Figure $2 A$ and $B$ ). Figure $2 C$ shows that $I_{T 1}$ amplitude was also significantly larger in females with than without AF. Moreover, Figure $2 \mathrm{D}$ shows that the higher incidence of $I_{\mathrm{Tl}}$ concurred with a higher incidence of spontaneous membrane depolarizations in female patients with AF. Because paroxysmal and long-standing persistent or permanent AF have been associated with different alterations in calcium homoeostasis, ${ }^{12}$ we compared their effects. As shown in Supplementary material online, Figure S4, women with paroxysmal and long-standing persistent or permanent AF displayed a similar increase in the $I_{\mathrm{TI}}$ frequency. However, the $I_{\mathrm{Ca}}$ density was reduced in males with long-standing persistent or permanent but not paroxysmal AF.

\subsection{Effect of sex on spontaneous SR calcium release}

To determine if the higher incidence of $I_{T I}$ and spontaneous membrane depolarizations were due to sex-specific differences in the susceptibility of the SR to release calcium spontaneously, we analysed the frequency and properties of calcium sparks in atrial myocytes from a subset of patients. This analysis showed interaction between AF and spark density $(P<0.001)$ as well as an interaction between AF plus sex and spark density $(P=0.037)$. In line with a differential interaction of female sex plus AF and calcium spark frequency and properties, the spark site density (Figure $3 A$ and $B$ ), the sparks/site (Figure $3 C$ ), the duration at half maximum (Figure 3D), and the full width at half maximum (Figure 3E) were all significantly higher in females with than without AF while no differences were observed in these features among males with and without AF. The calcium spark amplitude was not different among the different patient groups (Figure 3F).

\subsection{Effect of sex on the regulation of SR calcium uptake and content}

Figure $4 A$ and $B$ shows that the caffeine releasable SR calcium load, which regulates spontaneous $S R$ calcium release, was unaffected by sex and atrial rhythm and that sex had no effect on the calcium load in patients without or with AF. Analyses of the expression of the SR calcium pump SERCA2a and the $\mathrm{Na}-\mathrm{Ca}$ exchanger protein $\mathrm{NCX}-1$ that regulate the cytosolic calcium level showed that SERCA2a expression was significantly higher in women without than with AF (Figure 4C). NCX-1 expression was not significantly different among patients with and without $A F$, and sex did not affect the expression in either group (Figure 4D). Analysis of the expression and phosphorylation of PLB, which regulates SERCA2a activity, showed a significantly higher level of PLB in men without than with AF. This was also true for PLB phosphorylation at t17, while differences in phosphorylation at s16 were not significant (Supplementary material online, Figure S5). Interestingly expression of the SR calcium buffering protein Csq-2, which dampens RyR2 activity, was significantly reduced in both males and females with AF (Figure $5 \mathrm{~A}$ and $B$ ), Moreover, ratiometric immunofluorescent analysis of the Csq-2 distribution at the RyR2 clusters (Supplementary material online, Figure S6) showed that Csq-2 was highest at the sarcolemma in both male and female atrial myocytes (Figure $5 C$ and $D$ ) and there was a significant interaction between $\mathrm{AF}$ and the Csq-2/RyR2 ratio $(P<0.001)$.

\subsection{Effect of sex on RyR2 phosphorylation}

To determine if the increase in calcium sparks in women with AF could be caused by differential phosphorylation of the RyR2, we measured the density and phosphorylation state of individual RyR2 
A

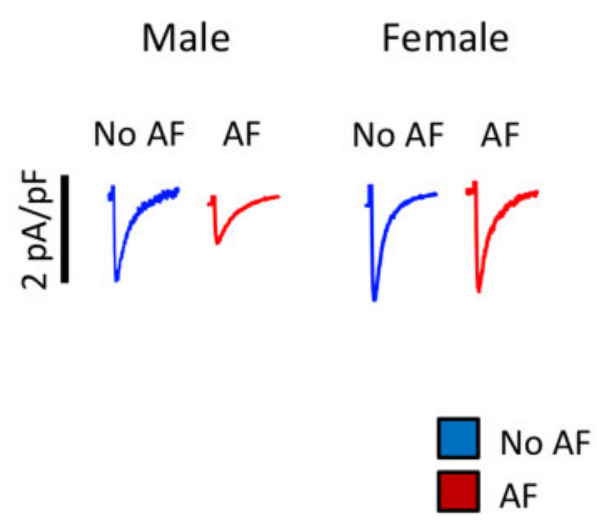

C

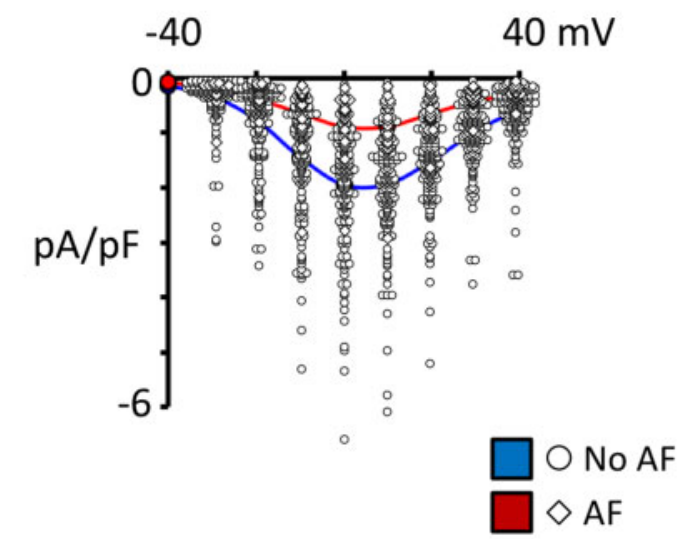

D

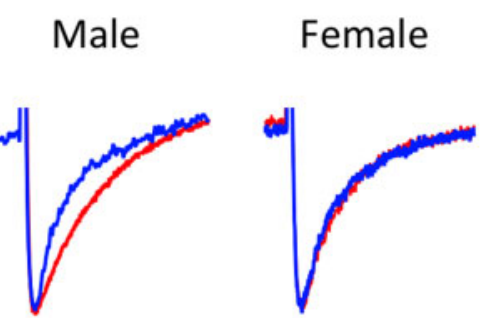

B

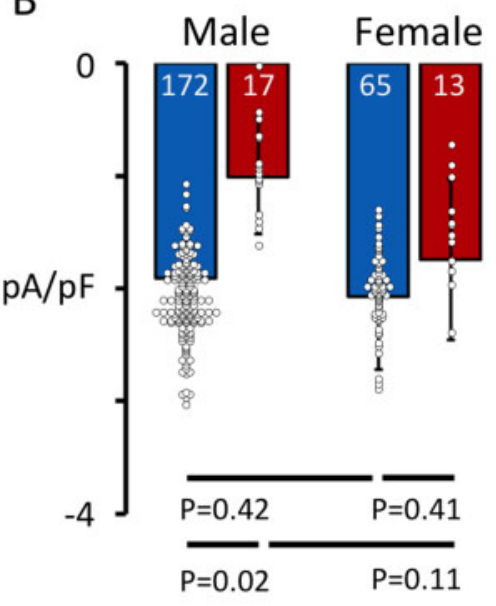

Female

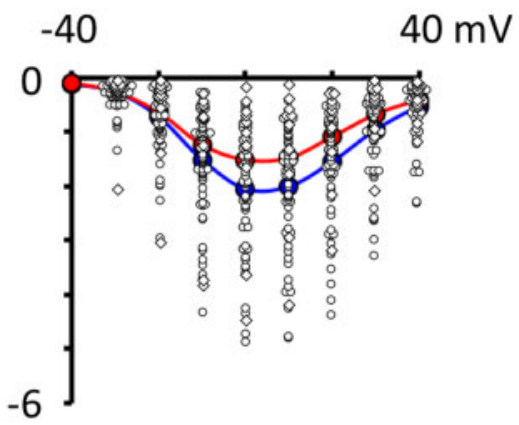

Figure I Effect of sex on the L-type calcium current amplitude and properties. (A) Representative $I_{C a}$ recordings from four patient groups of male (left traces) and female patients (right traces), without $A F$ (blue traces) and with $A F$ (red traces). (B) Mean $I_{C a}$ densities in the four groups. (C) Current-voltage curves for male and female patients with no AF or with AF. (D) Superimposed $I_{C_{a}}$-tracings are shown on the left and time constants for fast $I_{C a}$ inactivation are shown on the right. Values were analysed and corrected for the clinical factors marked as confounders in Table 1 using a linear regression model. $P$ values are given for significant differences between bars. Number of patients is given for each bar.

clusters at s2808 and s2814 (Supplementary material online, Figure S3 for details). This analysis showed that similar to Csq-2, RyR2 density was higher at the sarcolemma, but there were no differences in the RyR2 density among men and women with or without AF (Figure 6A and $B)$. However, linear regression analysis showed a significant interaction between AF plus sex and the s2808/RyR2 $(P=0.018)$ ratio with a strong increase in RyR2 phosphorylation at $s 2808$ in women but not in men with AF (Figure 6C and D). Separate images of total and 
A

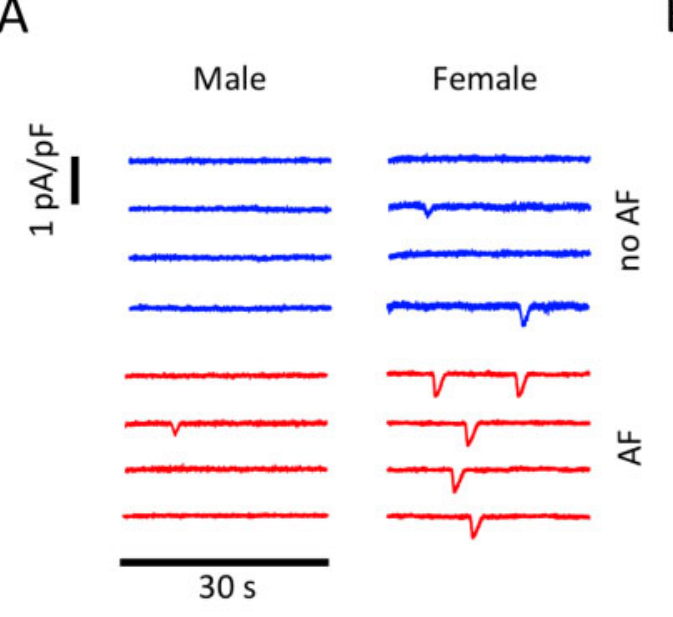

C

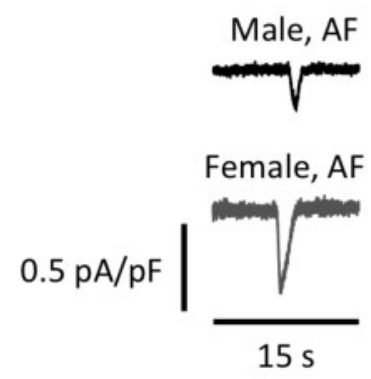

D

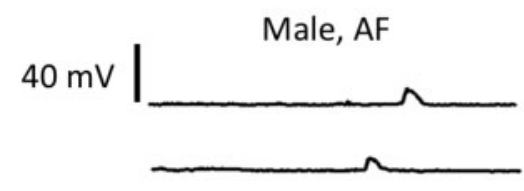

Female, AF

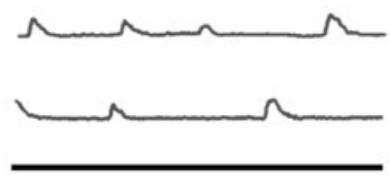

$30 \mathrm{~s}$
B
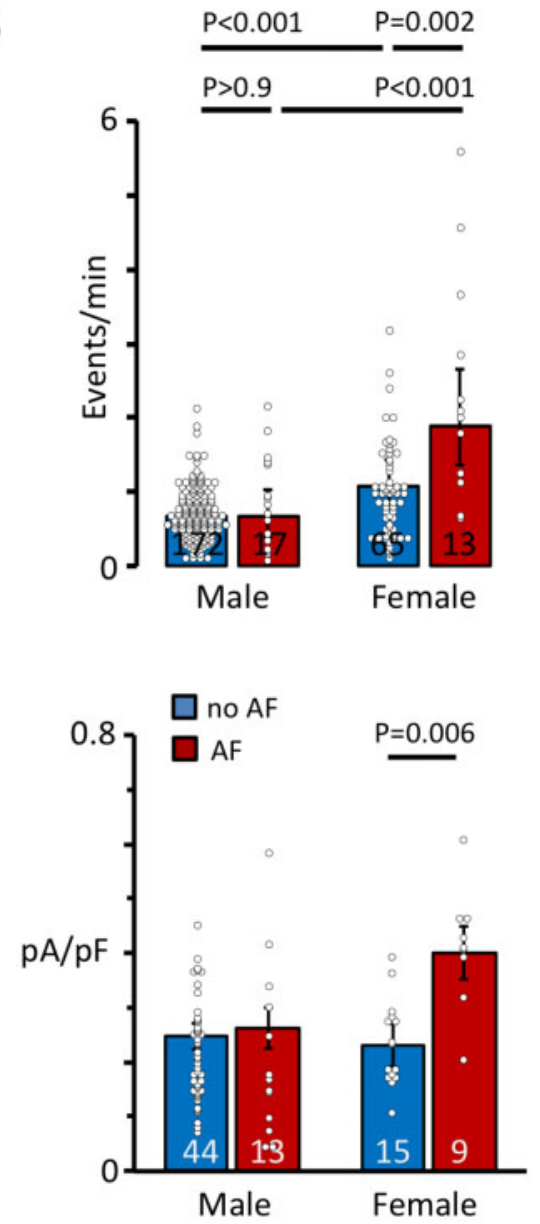

Figure 2 Effect of sex on the $I_{T I}$ frequency and spontaneous membrane depolarizations. (A) Representative recordings of $I_{T /}$ currents from the same patients as in Figure 1 divided into male (left traces) and female patients (right traces). (B) Mean $I_{T 1}$ frequencies. Values in $(A)-(B)$ were analysed and corrected for the clinical factors marked as confounders in Table 1 using a linear regression model. (C) Representative recordings of $I_{T I}$ amplitudes in a male and a female patient with AF. Mean amplitudes are shown on the right. (D) Spontaneous membrane depolarizations recorded in male and female patients with AF. The mean frequency is shown on the right. Statistical significance was determined in $(C)-(D)$ using an unadjusted regression model. $P$-values are given for significant differences between bars. Number of patients is given for each bar.

s2808 phosphorylated RyR2 are shown in Supplementary material online, Figure S7. RyR2 phosphorylation at s2814 was barely detectable in resting human atrial myocytes and there were no differences in s2814 phosphorylation, measured as the s2814/RyR2 ratio, between myocytes from nine female patients without $(0.21 \pm 0.04)$ and four with AF $(0.16 \pm 0.05)$. 
A
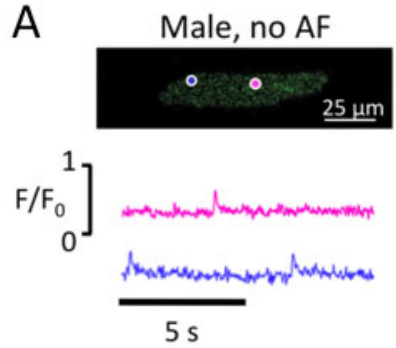

$5 \mathrm{~s}$
Male, AF
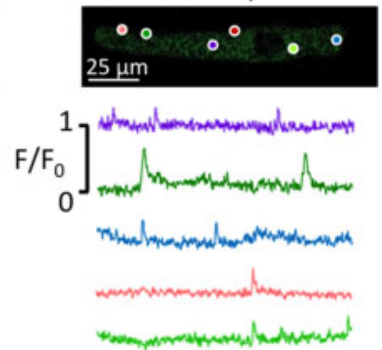

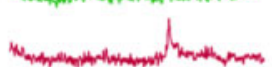

Female, no AF

\section{$25 \mu \mathrm{m}$}

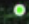

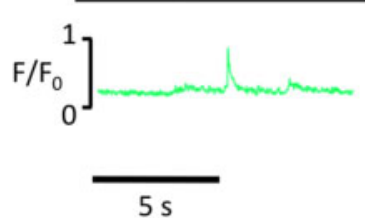

$5 \mathrm{~s}$
Female, AF
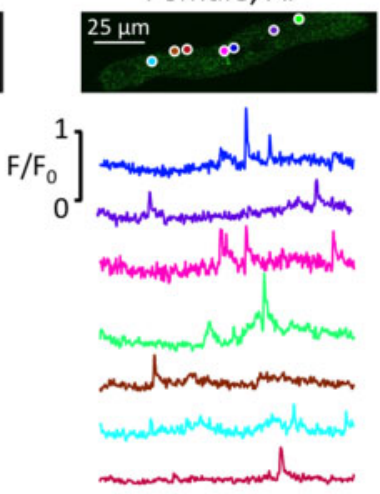

B

$$
\begin{array}{lll}
P=0.12 & P=0.001 \\
P=0.7 & P<0.001
\end{array}
$$

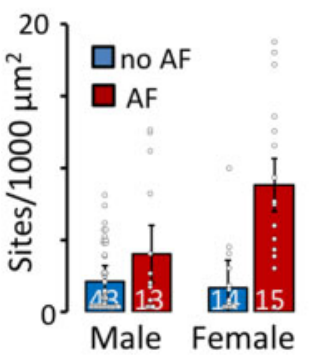

C
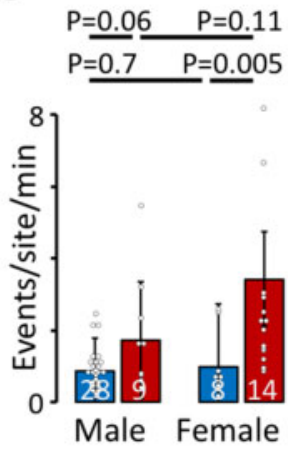

D
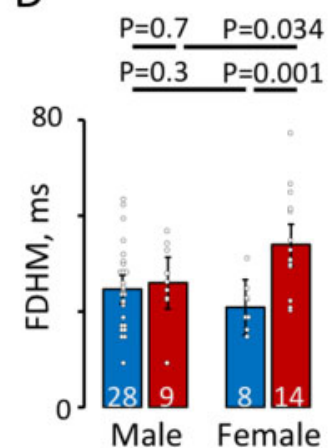

$E$
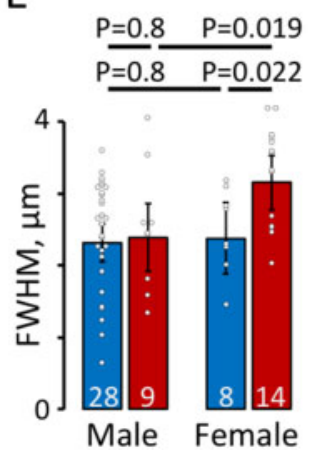

$\mathrm{F}$

$P=0.7 \quad P=0.9$

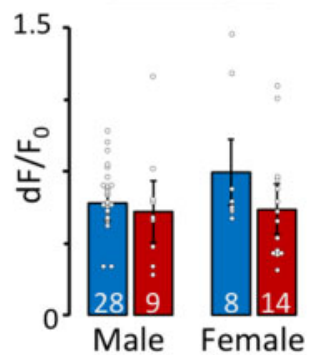

Figure 3 Effects of sex on calcium spark frequency and properties. (A) Images of human atrial myocytes from patients without (no AF) and with AF. Calcium spark sites are indicated with circles and calcium signals for each site are shown below. (B) Density of spark sites. (C) Sparks per site. (D) Spark duration at half maximum. (E) Spark width at half maximum $(F)$ Spark amplitude. Statistical significance was determined using an unadjusted linear regression model. $P$-values are given for significant differences between bars. Number of patients is given for each bar.

\subsection{Translation of sex-specific differences in calcium homoeostasis}

To determine if the observed sex-specific differences in calcium homoeostasis could be extended to pharmacological treatments directed towards a control of spontaneous calcium release, we focused on membrane receptors that are expected to exert a stronger modulation of s 2808 phosporylation near the sarcolemma as observed in Figure 6C and $D$. First, we used pharmacological manipulation of $A_{2 A} R s$, which has been shown to modulate spontaneous calcium release in myocytes from patients with $A F .{ }^{10}$ Figure $7 A$ shows that $A_{2 A} R$ activation with CGS21680 increased the $I_{T I}$ frequency in all patient groups and prevention of $A_{2 A} R$ activation with ADA reduced it in all groups (Figure $7 B$ ). This effect was most pronounced in females and especially in females with AF where it reduced the $I_{T 1}$ frequency below the level observed in patients without AF.

Secondly, analysis of patients that had been treated with beta-adrenergic receptor blockers, which might reduce RyR2 phosphorylation at s $2808,{ }^{18}$ revealed that this treatment reduced the $I_{T \text { I }}$ frequency dramatically in females with AF to levels observed in patients without AF (Figure 7C), and linear regression analysis showed a significant interaction between beta-blocker treatment and $I_{\mathrm{TI}}$ frequency $(P<0.001)$ as well as a combined interaction of beta-blocker treatment plus AF with $I_{\mathrm{TI}}$ frequency $(P=0.009)$.
In contrast to these results, $I_{\mathrm{Ca}}$ density was not affected by treatment of myocytes with ADA $(-1.87 \pm 0.24$ vs. $-1.87 \pm 0.20 \mathrm{pA} / \mathrm{pF})$ or in patients treated with beta-blockers $(-2.03 \pm 0.13$ vs. $-1.91 \pm 0.08 \mathrm{pA} / \mathrm{pF})$. Furthermore, none of these compounds were able to rescue the observed decrease in $I_{\mathrm{Ca}}$ density in male patients with AF where ADA decreased $I_{\mathrm{Ca}}$ density by $17 \pm 10 \%$ and the $I_{\mathrm{T}}$ frequency was $37 \pm 11 \%$ smaller in patients treated with beta-blockers.

\section{Discussion}

\subsection{Main findings}

This study is the first to analyse a large series of 267 consecutive patients in order to analyse the specific influence of sex on calcium homoeostasis in human atrial myocytes, taking into account confounding effects of other clinical factors that might affect the $I_{\mathrm{Ca}}$ density or the incidence of $I_{T /}$ currents. These analyses document that the $I_{C_{a}}$ density is only diminished significantly in males with AF while a higher incidence of both $I_{\mathrm{TI}}$ and spontaneous membrane depolarizations is observed almost exclusively in females and is exacerbated in those with AF. Analysis of the underlying mechanisms revealed that females with AF showed a differential increase in spontaneous calcium release from the SR linked to a higher level of RyR2 phosporylation at S2808. Furthermore, prevention of $A_{2 A} R$ activation with ADA or treatment of patients with beta-blockers 
A

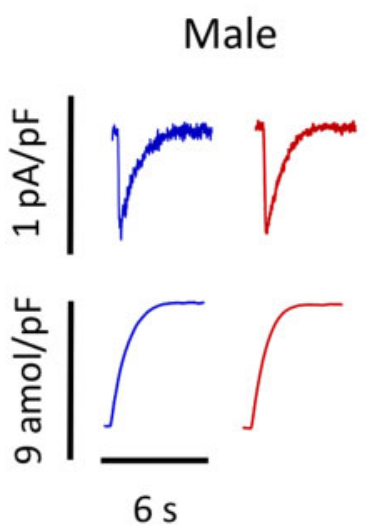

C

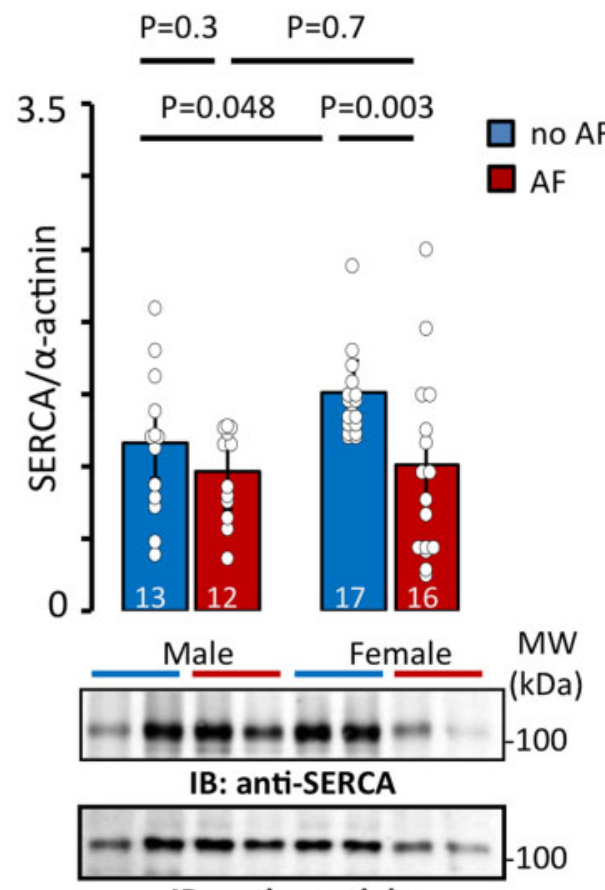

IB: anti- $\alpha$-actinin
B
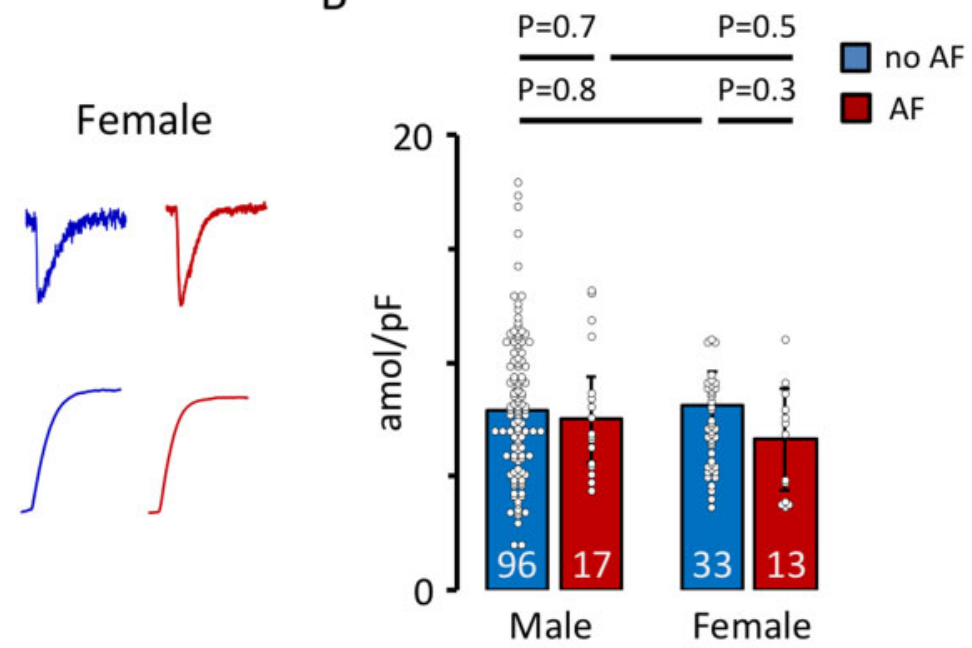

D

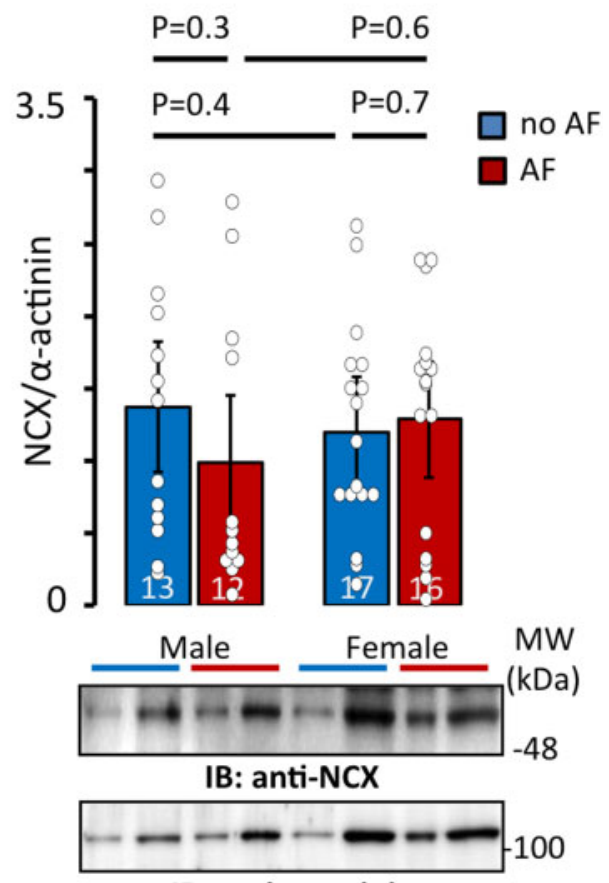

IB: anti- $\alpha$-actinin

Figure 4 Effect of sex on SR calcium load and uptake. (A) Representative caffeine-induced transient inward NCX currents (top) and their time-integral (bottom) from a male and a female patient without AF (left) and with AF (right). (B) Caffeine releasable SR calcium load estimated from the time-integral of the caffeine-induced current. Values are corrected for the clinical factors marked as confounders in Table 1 using a linear regression model. ( $C$ ) SERCA2a protein expression. (D) NCX-1 protein expression. Densitometry quantification of protein levels is shown in the upper panels and representative western blots in the lower panels. Protein levels were normalized to $\alpha$-actinin. $P$-values are given for differences between bars. Statistical significance was determined using an unadjusted linear regression model. Number of patients is given for each bar.

was able to reduce the $I_{\mathrm{TI}}$ frequency in females with AF to levels observed in patients without AF.

\subsection{Sex and L-type calcium current}

While the effects of mitral valve disease ${ }^{16}$ ageing, ${ }^{19}$ or left ventricular function ${ }^{20}$ on $I_{\mathrm{Ca}}$ density or $I_{\mathrm{T}}$ frequency have been assessed in human atrial myocytes, studies addressing sex-related effects on calcium handling have been limited to mammalian models. ${ }^{21-23}$
Thus, elevation of testosterone levels has been shown to reduce $I_{\text {Ca }}$ in guinea-pig ventricular myocytes ${ }^{23}$ pointing to potential sex-specific differences in the $I_{C a}$ density in healthy animals. In contrast to this, we found that sex showed no interaction with $I_{\mathrm{Ca}}$ density in human atrial myocytes when the confounding effects of the clinical factors from Table 1 were taken into account. Pairwise comparison of the patient groups did, nevertheless, show a significant reduction of the $I_{\mathrm{Ca}}$ density in men but not in women. Thus, the significant effect 
A

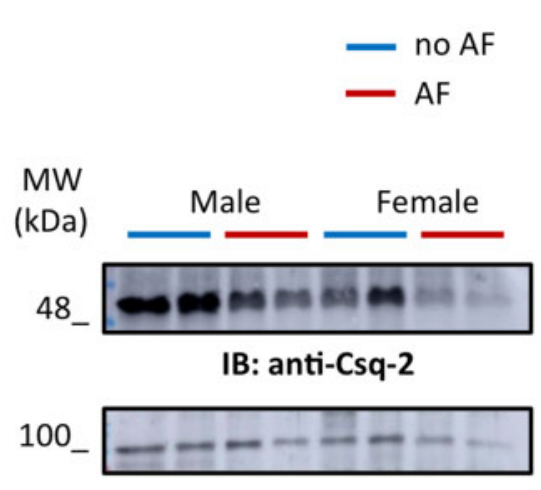

IB: anti- $\alpha$-actinin
B

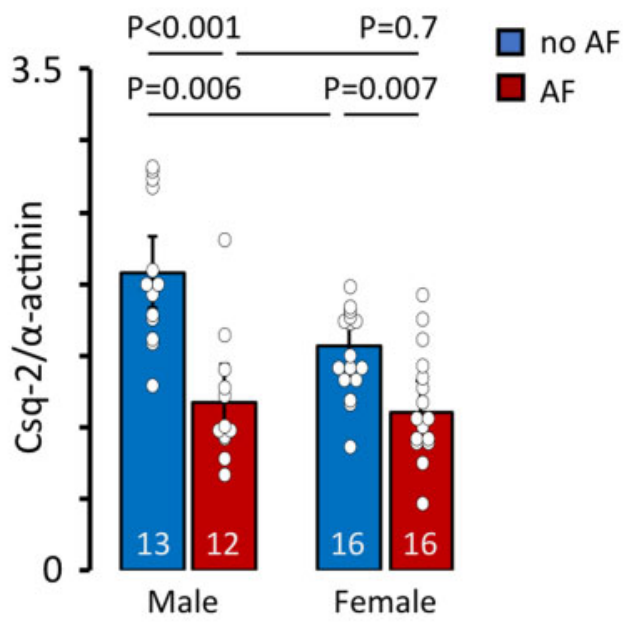

Female
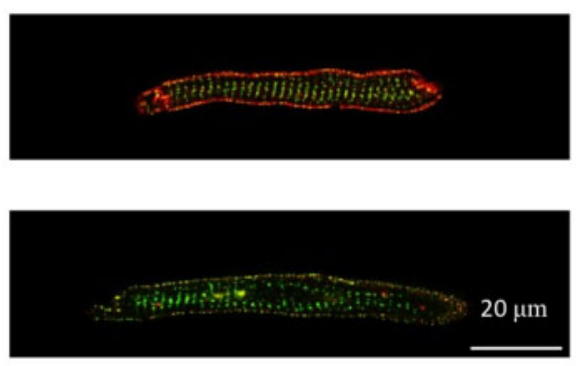

no AF (49)

AF (8)

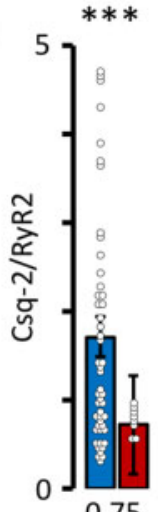

0.75

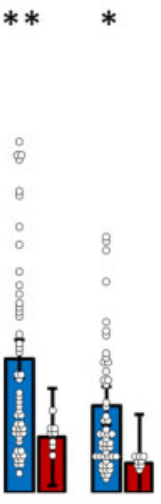

1.5

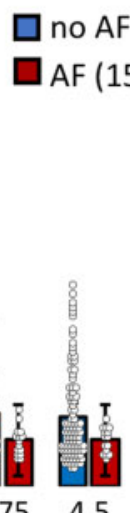

Figure 5 Effect of sex on the expression and distribution of Csq-2. (A) Representative western blots of Csq- 2 and $\alpha$-actinin. (B) Densitometry quantification of Csq-2expression normalized to $\alpha$-actinin. The number of patients is indicated for each bar. $P$-values are given above bars. (C) Overlay of fluorescently labelled RyR2 (in green) and Csq-2 (in red). (D) Csq-2/RyR2 intensity ratios measured at different distances from the sarcolemma (given below bars in $\mu \mathrm{m}$ ). Atrial myocytes from No AF patients had higher ratios than those from AF for females $(P=0.02)$ but not for males $(P=0.06)$. Statistical significance was determined using an unadjusted linear regression model. Statistical differences between pairs of $A F$ and noAF are indicated with $* * * P<0.001$, $* * P<0.01, * P=0.05$. Number of experiments is given in parentheses.

of $\mathrm{AF}$ on $I_{\mathrm{Ca}}$ density reported in most studies of mixed sex, ${ }^{11,24}$ is possibly contributed by the male patients in the study population. Sex had no differential effect on the shape of the current-voltage relationship in patients with AF or in those without AF, suggesting that sex does not affect the gating properties of the L-type calcium channel. However, the kinetics of $I_{\mathrm{Ca}}$ inactivation was significantly slower in male than in female patients with AF. Considering that calcium release from the SR modulates the fast time constant for $I_{\mathrm{Ca}}$ inactivation, the faster inactivation in females than males with AF might be due to a higher lability of SR calcium release in these patients (see below) or because there is only an insignificant loss of L-type calcium current in females with $A F$, whereas the smaller $I_{\mathrm{Ca}}$ amplitude in males with $A F$ is expected to slow inactivation in this group. While the observed reduction in $I_{\mathrm{Ca}}$ amplitude might be due to reduced expression of L-type calcium channels previous 
A

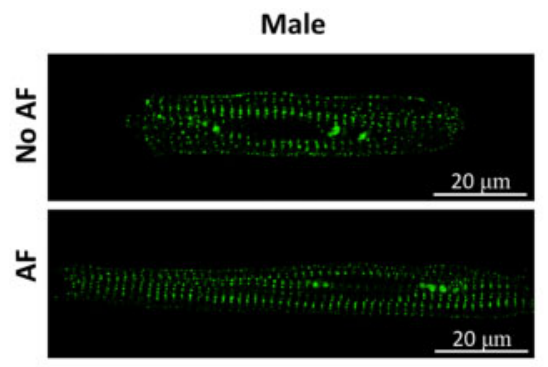

B

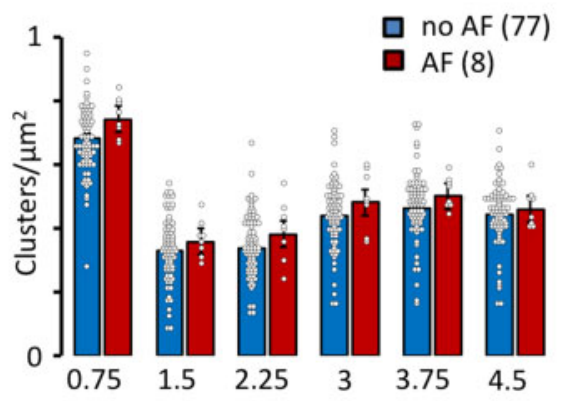

C

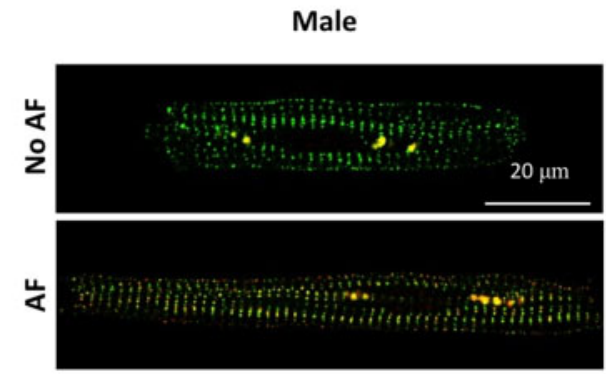

D
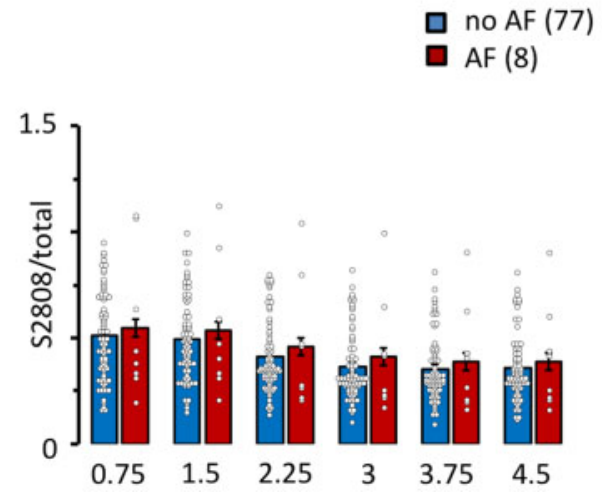
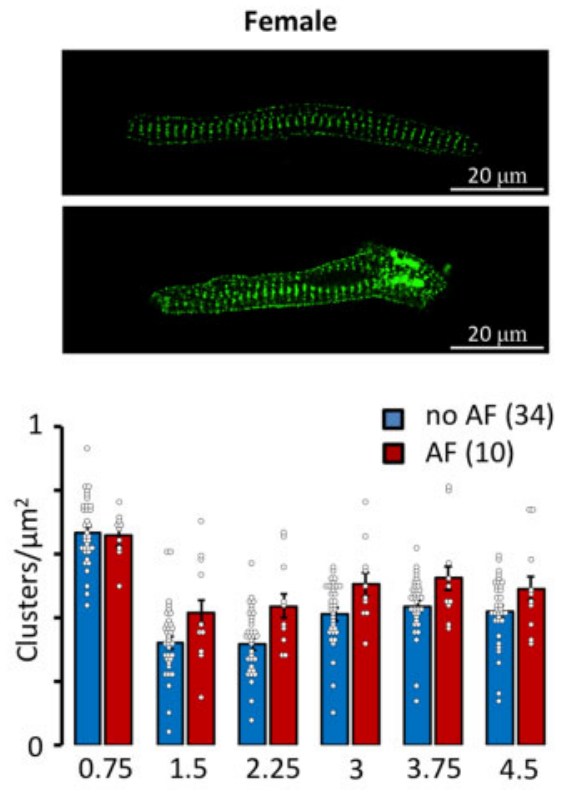

Female
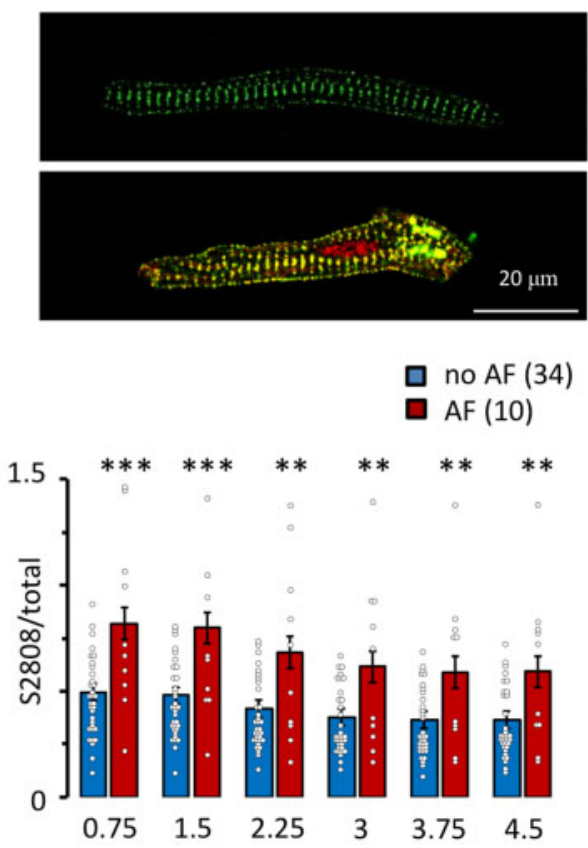

Figure 6 Effect of sex on the density and phosphorylation of the RyR2. (A) Immunoflourescent labelling of the RyR2. (B) Mean density of RyR2 clusters. (C) Overlay of total RyR2 (in green) and s2808 phosphorylated RyR2 (in red). (D) Mean s2808 phosphorylated RyR2 measured as the fluorescence intensity ratio (s2808/RyR2) for all RyR2 clusters at different distances from the sarcolemma (given below bars in $\mu \mathrm{m}$ ). Statistical significance was determined using an unadjusted linear regression model. Significant differences between pairs of bars are indicated with $* * * P<0.001, * * P<0.01$. Number of experiments is given in parentheses.

findings are ambiguous with some studies reporting a reduction ${ }^{25,26}$ while others found no changes. ${ }^{27}$ Here, we found that the smaller $I_{\mathrm{Ca}}$ in males with AF did not coincide with a reduction in the density of L-type calcium channels, pointing to other factors, such as oxidative stress $^{28}$ or $\operatorname{Cav} \beta 2^{29}$ as potential modulators of $I_{C a}$ amplitude in males with AF.

\subsection{Sex and spontaneous SR calcium release}

When taking into account the confounding effects of other clinical factors, both sex and sex plus AF showed significant interactions with the $I_{T 1}$ frequency. Thus, male sex barely affected the $I_{T 1}$ frequency when comparing patients with and without $A F$, while the $I_{T 1}$ frequency was higher in 

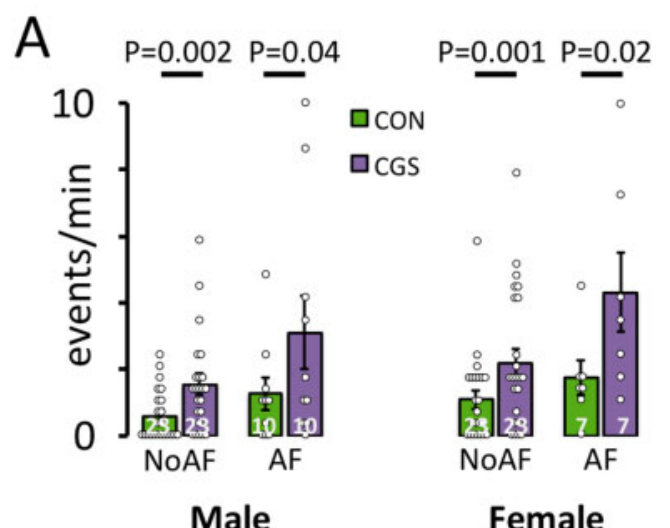

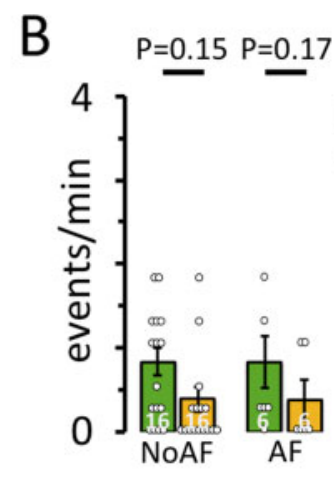

Male

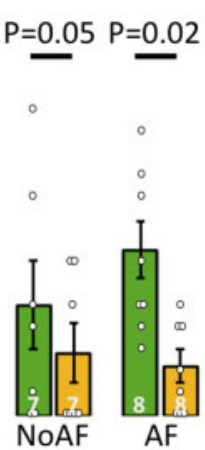

Female

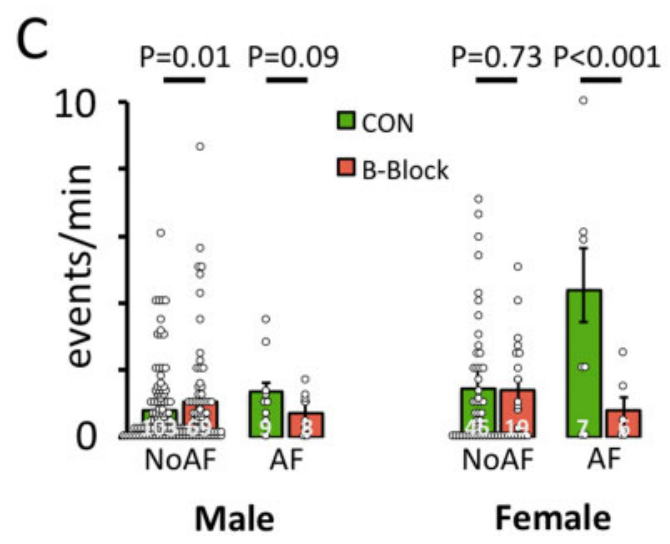

Figure $7 A_{2 A} R$ inhibition or treatment with beta-blockers reduces the $I_{T 1}$ frequency in females with AF. (A) Effect of $A_{2 A} R$ activation with CGS21680 on the $I_{T l}$ frequency. (B) Prevention of $A_{2 A} R$ activation by treating myocytes with ADA reduces the $I_{T l}$ frequency in females with AF to levels observed in patients without AF. (C) Treatment of patients with beta-blockers (B-Block) reduces the $I_{T /}$ frequency in female patients with $A F$ to levels in patients without AF. Data were analysed using an unadjusted regression model. P-values for paired comparison of pharmacological treatment and control are given above bars. Number of patients is given for each bar.

females than males without $A F$, and it increased dramatically in women with AF. Additionally, the $I_{\mathrm{TI}}$ amplitude increased significantly in women with $A F$, increasing the ability of the $I_{T 1}$ to induce spontaneous membrane depolarizations. In line with this, the incidence of spontaneous membrane depolarizations in AF patients was twice as high in females as in males. In accordance with a role for female sex hormones in the regulation of spontaneous membrane depolarizations in females, ovariectomy has been shown to induce calcium-handling disturbances and a higher incidence of early afterdepolarizations in guinea-pig myocytes. ${ }^{21}$ This would agree with a higher incidence of membrane depolarizations in elderly female patients with $A F$, but does not explain why spontaneous calcium release is lower in males and further studies are required to elucidate how sex hormones affect calcium homoeostasis in human atrial myocytes.

Analysis of the mechanisms underlying the higher $I_{T I}$ frequency in our human atrial myocytes demonstrated that the number of calcium spark sites was significantly increased in female but not in male patients with $\mathrm{AF}$ and this concurred with wider and longer lasting sparks, facilitating their fusion into calcium waves, triggering electrogenic $\mathrm{Na}-\mathrm{Ca}$ exchange and membrane depolarizations. The higher density of calcium spark sites in women with AF was not caused by SR calcium overload since neither sex nor AF had any significant effect on the caffeine releasable SR calcium content. The latter is in line with previous reports, $9,10,15$ and western blot analysis revealed that SERCA expression was in fact significantly higher in female patients without AF, which on the other hand could explain the larger spark amplitude observed in females without AF. There were no significant differences in NCX-1 or PLB expression when comparing women with and without AF, and PLB phosphorylation at ser16 or thr17 was also similar, discarding effects on SR calcium uptake. Potentially, SR calcium uptake could also be increased by a lower sarcolipin expression in $A F,{ }^{20,30}$ but this notion is not supported by our measurements of unchanged SR calcium load in both male and female patients with AF.

However, measurement of Csq-2 levels and s2808 phosphorylation at each RyR2 cluster showed significantly lower Csq-2 levels in female patients with $A F$ than without $A F$, which may contribute to increase the spark frequency ${ }^{31,32}$ in those with AF. The same is true for the higher s2808 phosphorylation, observed in myocytes from women with AF, which has previously been associated with AF in populations of mixed sex. $^{10,12,14,15}$ The fact that increased $s 2808$ phosphorylation is only observed in women with AF, also suggests that caution should be taken when interpreting analyses of s2808 phosphorylation in mixed populations of male and female patients with AF because the increase and 
statistical significance may vary according to the fraction of female patients, especially when the study population is small. Indeed, some studies with small populations find a significant difference in s2808 phosphorylation between patients with and without $A F,{ }^{10,12,14,15}$ while others do $_{\text {not. }}{ }^{13}$

In a previous study of a mixed sex population, paroxysmal AF has also been associated with elevation of spontaneous calcium release-induced membrane depolarizations. ${ }^{12}$ In the present study, paroxysmal AF was initially included in the analysis but the multivariate statistical analysis of $I_{\mathrm{Ca}}$ density and $I_{T l}$ frequency in patients with paroxysmal AF did not afford statistical support for interactions with sex and atrial rhythm, and we therefore chose not to include this patient group in the final analysis. It should nevertheless be pointed out that post-test comparison showed that the $I_{T}$ frequency in female patients with paroxysmal and long-standing persistent or permanent AF was comparable and significantly higher than the frequency recorded in male patients and in female patients without AF.

\subsection{Study limitations}

A limitation of this study is the modest yield of healthy human atrial myocytes, which limits the number of electrophysiological experiments that can be carried out for each patient. Therefore, measurements from individual patients might not always be representative, although we previously found that variation among samples from the same patient was significantly smaller than variation among samples from different patients in a study population. ${ }^{9}$

Moreover, we only used human right atrial specimens in this study, and cannot rule out that not all of the present findings apply to the left atrium also. However, extraction of left atrial tissue samples for myocyte isolation is ethically justifiable in a limited number of cases such as mitral valve surgery where the left atrium is usually diseased and dilated and has been reported to disturb the calcium homoeostasis. ${ }^{16}$ Thus, electrophysiological analysis of myocytes from patients with diseased or nearly normal atria is only realistic for the right atrium.

Finally, further studies with a larger number of patients are required to reach firm conclusions on some of the effects of sex in patients with and without AF where the effect size is smaller. For example, betablocker treatment is expected to reduce the impact of sex on s2808 phosphorylation in patients without AF but a larger sample size is necessary to achieve sufficient statistical power to test this. The same is true for the influence of sex on the effects in paroxysmal AF, which has previously been associated with alterations in calcium homoeostasis that are different from patients with long-standing persistent or permanent AF. ${ }^{12}$ Similarly, pairwise comparisons showed a reduced $I_{C a}$ density in males with $\mathrm{AF}$ even though there were no interaction between sex, $\mathrm{AF}$ and $I_{\mathrm{Ca}}$ in this study, which is possibly due to the small number of patients with AF compared the number of patients without AF.

\subsection{Clinical relevance, translation, and conclusions}

This is the first study to examine the effect of sex on the intracellular calcium homoeostasis in atrial myocytes from a large series of patients that takes into account confounding effects of common risk factors and concurring cardiovascular disease. Our findings demonstrate that sex has differential effects on the $I_{\mathrm{Ca}}$ density and the $I_{\mathrm{T}}$ frequency in atrial myocytes from patients with $A F$, suggesting that therapeutical approaches to prevent AF may be optimized by taking into account the observed differences in calcium homoeostasis between males and females. Thus, a reduction in $I_{\mathrm{Ca}}$ density previously associated to $\mathrm{AF}$ in populations of mixed sex is more prominent in males with AF, suggesting that therapies normalizing L-type calcium channel activity might be more efficient in males and that it may be worthwhile to consider L-type calcium channels as a therapeutical target in males with AF. In this regard, the present findings show that beta-blocker treatment is unable to rescue $I_{\mathrm{Ca}}$ in males with $\mathrm{AF}$, pointing to alternative targets, such as $\operatorname{Cav} \beta 2^{29}$ that is capable of restoring $I_{\mathrm{Ca}}$ in diseased hearts. On the other hand, therapies targeting mechanisms that modulate spontaneous calcium release and membrane depolarizations ${ }^{10,13,15,18}$ are expected to be efficient in females with $A F$ where we observed a high incidence of $I_{\mathrm{Tl}}$ currents and membrane depolarization, but unsuccessful in males where the incidence of these arrhythmogenic events is low even in patients with AF. Indeed, we here show that prevention of $A_{2 A} R$ activation was capable of reducing the high incidence of $I_{T S}$ in females with AF to levels observed in patients without AF. Also, in line with a beneficial effect of reducing receptor-mediated hyperphosphorylation of the RyR2, we demonstrate that female $\mathrm{AF}$ patients treated with beta-blockers had an $I_{T I}$ frequency similar to that observed in patients without AF.

It should nevertheless be kept in mind that the patients treated with beta-blockers did have AF in spite of a low $I_{T I}$ frequency, which might suggest that an elevated $I_{T \text { I }}$ frequency is not the cause but rather a consequence of AF. However, patients with genetic risk variants at chromosome $4 \mathrm{q} 25$ that increase the risk of AF present elevation of the $I_{\mathrm{T}}$ frequency that precedes the development of AF. ${ }^{16}$ This in turn suggests that $I_{T \mid}$ suppression with beta-blockers might prevent or delay the onset of $A F$ in patients prone to present calcium release-induced ectopic activity. By contrast, beta-blockers may be less effective in patients where the underlying mechanism is atrial fibrosis ${ }^{33}$ or dysregulated ion channel activity that is unrelated to spontaneous calcium release. ${ }^{34}$

In summary, our findings demonstrate that further studies aiming to discover pharmacological targets for restoration of the L-type calcium current in male patients with AF are warranted while therapies aiming to normalize RyR2 phosphorylation may be useful to prevent excessive SR calcium release in females with AF.

\section{Supplementary material}

Supplementary material is available at Cardiovascular Research online.

\section{Authors' contributions}

A.H.-M., C.T., V.J.-S., A.L., H.G.-M., F.C., and L.H.-M. designed the research. A.H.-M., C.T., V.J.-S., A.L., H.G.-M., H.C., C.N.-C., S.C., and P.I.-C. performed the research. A.H.-M., C.T., V.J.-S., A.L., H.G.-M., H.C., C.N.C., S.C., P.I.-C., I.B., R.B., E.R.-D., E.R.-F., X.V., F.C., J.C., and L.H.-M. analysed the data. A.H.-M., C.T., V.J.-S., F.C., J.C., and L.H.-M. wrote the article.

\section{Acknowledgements}

The collaboration of the Cardiac Surgery Department at Hospital de la Santa Creu i Sant Pau is greatly appreciated.

Conflict of interest: none declared.

\section{Funding}

This work was supported by grants from The Spanish Ministry of Science Innovation and Universities [SAF2017-88019-C3-1-R MICIU /AEI/ FEDER / 
UE] to L.H.-M. [SAF2017-88019-C3-2-R MICIU /AEI/ FEDER /UE] to R.B. and [ SAF2017-87349-R MICIU /AEI/ FEDER /UE] to F.C.; and from the Spanish Ministry of Health and Consume, ISCIII, CIBERCV [CB16/11/00276] and Fondo Europeo de Desarrollo Regional (FEDER) to J.C., Fundació Marato TV3 [20152030/31] to L.H.-M./F.C. Also supported by ISC III [PIE14/ 00034] and IWT [SBO-140028] to F.C., a PhD grant [FPU/01250] to S.C., a PERIS grant from Generalitat de Catalunya to A.L. and [SGR2017-1769] to L.H.-M.

\section{Data availability}

The data underlying this article will be shared on reasonable request to the corresponding author.

\section{References}

1. Ko D, Rahman F, Schnabel RB, Yin X, Benjamin EJ, Christophersen IE. Atrial fibrillation in women: epidemiology, pathophysiology, presentation, and prognosis. Nat Rev Cardiol 2016;13:321-332.

2. Gowd BM, Thompson PD. Effect of female sex on cardiac arrhythmias. Cardiol Rev 2012;20:297-303.

3. Humphries KH, Kerr CR, Connolly SJ, Klein G, Boone JA, Green M, Sheldon R, Talajic M, Dorian P, Newman D. New-onset atrial fibrillation: sex differences in presentation, treatment, and outcome. Circulation 2001;103:2365-2370.

4. Benjamin EJ, Wolf PA, D'Agostino RB, Silbershatz H, Kannel WB, Levy D. Impact of atrial fibrillation on the risk of death: the framingham heart study. Circulation 1998;98: 946-952.

5. Wijffels MC, Kirchhof C], Dorland R, Allessie MA. Atrial fibrillation begets atrial fibrillation. A study in awake chronically instrumented goats. Circulation 1995;92:1954-1968.

6. Ravens U. Sex differences in cardiac electrophysiology. Can J Physiol Pharmacol 2018; 96:985-990.

7. Allessie M, Ausma J, Schotten U. Electrical, contractile and structural remodeling during atrial fibrillation. Cardiovasc Res 2002;54:230-246.

8. Goette A, Honeycutt C, Langberg JJ. Electrical remodeling in atrial fibrillation. Time course and mechanisms. Circulation 1996;94:2968-2974.

9. Hove-Madsen L, Llach A, Bayes-Genís A, Roura S, Font ER, Arís A, Cinca J. Atrial fibrillation is associated with increased spontaneous calcium release from the sarcoplasmic reticulum in human atrial myocytes. Circulation 2004;110:1358-1363.

10. Llach A, Molina CE, Prat-Vidal C, Fernandes J, Casado V, Ciruela F, Lluis C, Franco R, Cinca J, Hove-Madsen L. Abnormal calcium handling in atrial fibrillation is linked to up-regulation of adenosine a2a receptors. Eur Heart J 2011;32:721-729.

11. Van Wagoner DR, Pond AL, Lamorgese M, Rossie SS, McCarthy PM, Nerbonne JM. Atrial l-type ca2+ currents and human atrial fibrillation. Circ Res 1999;85:428-436.

12. Voigt N, Heijman J, Wang Q, Chiang DY, Li N, Karck M, Wehrens XH, Nattel S, Dobrev D. Cellular and molecular mechanisms of atrial arrhythmogenesis in patients with paroxysmal atrial fibrillation. Circulation 2014;129:145-156.

13. Neef S, Dybkova N, Sossalla S, Ort KR, Fluschnik N, Neumann K, Seipelt R, SchöNdube FA, Hasenfuss G, Maier LS. Camkii-dependent diastolic sr ca2+ leak and elevated diastolic ca2+ levels in right atrial myocardium of patients with atrial fibrillation. Circ Res 2010;106:1134-1144.

14. Vest JA, Wehrens XH, Reiken SR, Lehnart SE, Dobrev D, Chandra P, Danilo P, Ravens $U$, Rosen MR, Marks AR. Defective cardiac ryanodine receptor regulation during atrial fibrillation. Circulation 2005;111:2025-2032.

15. Voigt N, Li N, Wang Q, Wang W, Trafford AW, Abu-Taha I, Sun Q, Wieland T, Ravens U, Nattel S, Wehrens XH, Dobrev D. Enhanced sarcoplasmic reticulum $\mathrm{ca} 2+$ leak and increased na+-ca2+ exchanger function underlie delayed afterdepolarizations in patients with chronic atrial fibrillation. Circulation 2012;125:2059-2070.

16. Dinanian S, Boixel C, Juin C, Hulot JS, Coulombe A, Rucker-Martin C, Bonnet N, Le Grand B, Slama M, Mercadier JJ, Hatem SN. Downregulation of the calcium current in human right atrial myocytes from patients in sinus rhythm but with a high risk of atrial fibrillation. Eur Heart J 2008;29:1190-1197.

17. Herraiz-Martínez A, Llach A, Tarifa C, Gandía J, Jiménez-Sabado V, Lozano-Velasco E, Serra SA, Vallmitjana A, Vázquez Ruiz de Castroviejo E, Benítez R, Aranega A, Muñoz-Guijosa C, Franco D, Cinca J, Hove-Madsen L. The 4q25 variant rs13143308t links risk of atrial fibrillation to defective calcium homoeostasis. Cardiovasc Res 2019; 115:578-589.

18. Reiken S, Wehrens XH, Vest JA, Barbone A, Klotz S, Mancini D, Burkhoff D, Marks AR. Beta-blockers restore calcium release channel function and improve cardiac muscle performance in human heart failure. Circulation 2003;107:2459-2466.

19. Herraiz-Martínez A, Álvarez-García J, Llach A, Molina CE, Fernandes J, FerreroGregori A, Rodríguez C, Vallmitjana A, Benítez R, Padró JM, Martínez-González J, Cinca J, Hove-Madsen L. Ageing is associated with deterioration of calcium homeostasis in isolated human right atrial myocytes. Cardiovasc Res 2015;106:76-86.

20. Molina CE, Abu-Taha IH, Wang Q, Roselló-Díez E, Kamler M, Nattel S, Ravens U, Wehrens XHT, Hove-Madsen L, Heijman J, Dobrev D. Profibrotic, electrical, and calcium-handling remodeling of the atria in heart failure patients with and without atrial fibrillation. Front Physiol 2018;9:18.

21. Yang HY, Firth JM, Francis AJ, Alvarez-Laviada A, MacLeod KT. The effect of ovariectomy on intracellular calcium (ca2+) regulation in guinea pig cardiomyocytes. $\mathrm{Am} \mathrm{J}$ Physiol Heart Circ Physiol 2017;313:H1031-H1043.

22. Yaras N, Tuncay E, Purali N, Sahinoglu B, Vassort G, Turan B. Sex-related effects on diabetes-induced alterations in calcium release in the rat heart. Am J Physiol Heart Circ Physiol 2007;293:H3584-H3592.

23. Bai CX, Kurokawa J, Tamagawa M, Nakaya H, Furukawa T. Nontranscriptional regulation of cardiac repolarization currents by testosterone. Circulation 2005;112: 1701-1710.

24. Llach A, Molina CE, Fernandes J, Padro J, Cinca J, Hove-Madsen L. Sarcoplasmic reticulum and l-type ca2+ channel activity regulate the beat-to-beat stability of calcium handling in human atrial myocytes. J Physiol 2011;589:3247-3262.

25. Lai LP, Su MJ, Lin JL, Lin FY, Tsai CH, Chen YS, Huang SK, Tseng YZ, Lien WP. Down-regulation of L-type calcium channel and sarcoplasmic reticular $\mathrm{ca}(2+)$ ATPase mRNA in human atrial fibrillation without significant change in the mRNA of ryanodine receptor, calsequestrin and phospholamban: an insight into the mechanism of atrial electrical remodeling. J Am Coll Cardiol 1999;33:1231-1237.

26. Brundel IC, Henning RH, Tuinenburg AE, Deelman LE, Tieleman RG, Grandjean JG, van Gilst WH, Crijns HJ. Gene expression of proteins influencing the calcium homeostasis in patients with persistent and paroxysmal atrial fibrillation. Cardiovasc Res 1999;42:9.

27. Schotten UHH, Frechen D, Greiser M, Stellbrink C, Vazquez-Jimenez JF, Morano I, Allessie MA, Hanrath P. The l-type ca2+-channel subunits alpha1c and beta2 are not downregulated in atrial myocardium of patients with chronic atrial fibrillation. J Mol Cell Cardiol 2003;35:6.

28. Carnes CA, Janssen PM, Ruehr ML, Nakayama H, Nakayama T, Haase H, Bauer JA Chung MK, Fearon IM, Gillinov AM, Hamlin RL, Van Wagoner DR. Atrial glutathione content, calcium current, and contractility. J Biol Chem 2007;282:28063-28073.

29. Rusconi F, Ceriotti P, Miragoli M, Carullo P, Salvarani N, Rocchetti M, Di Pasquale E, Rossi S, Tessari M, Caprari S, Cazade M, Kunderfranco P, Chemin J, Bang M-L, Polticelli F, Zaza A, Faggian G, Condorelli G, Catalucci D. Peptidomimetic targeting of $\operatorname{cav} \beta 2$ overcomes dysregulation of the l-type calcium channel density and recovers cardiac function. Circulation 2016;134:534-546.

30. Shanmugam MM, Gao S, Severac-Bastide R, Fischmeister R, Babu GJ. Decreased sarcolipin protein expression and enhanced sarco(endo)plasmic reticulum ca2+ uptake in human atrial fibrillation. Biochem Biophys Res Commun 2011:410:4.

31. Glukhov AV, Kalyanasundaram A, Lou Q, Hage LT, Hansen BJ, Belevych AE, Mohler PJ, Knollmann BC, Periasamy M, Gyorke S, Fedorov VV. Calsequestrin 2 deletion causes sinoatrial node dysfunction and atrial arrhythmias associated with altered sarcoplasmic reticulum calcium cycling and degenerative fibrosis within the mouse atrial pacemaker complex1. Eur Heart J 2015;36:686-697.

32. Lou Q, Belevych AE, Radwański PB, Liu B, Kalyanasundaram A, Knollmann BC, Fedorov VV, Györke S. Alternating membrane potential/calcium interplay underlies repetitive focal activity in a genetic model of calcium-dependent atrial arrhythmias. J Physiol 2015;593:1443-1458.

33. Reil J-C, Hohl M, Selejan S, Lipp P, Drautz F, Kazakow A, Münz BM, Müller P, Steendijk P, Reil G-H, Allessie MA, Böhm M, Neuberger H-R. Aldosterone promotes atrial fibrillation. Eur Heart J 2012;33:2098-2108.

34. Caballero R, de la Fuente MG, Gómez R, Barana A, Amorós I, Dolz-Gaitón P, Osuna L, Almendral J, Atienza F, Fernández-Avilés F, Pita A, Rodríguez-Roda J, Pinto Á, Tamargo J, Delpón E. In humans, chronic atrial fibrillation decreases the transient outward current and ultrarapid component of the delayed rectifier current differentially on each atria and increases the slow component of the delayed rectifier current in both. J Am Coll Cardiol 2010;55:2346-2354.

\section{Translational perspective}

Statistical analysis taking into account confounding effects of concurrent disease, risk factors, and treatments revealed differential sex-dependent alterations of the calcium homoeostasis in AF. The analysis suggests that suppression of calcium release-induced membrane depolarizations with adenosine receptor antagonists may be efficient in women with AF only while therapies aiming to restore L-type calcium current may be more efficient in males with AF. 